\title{
Isotopenanreicherung bei Lithium durdh elektrolytische Überführung in geschmolzenem Lithiumbromid
}

\author{
Von Arnold Lundén \\ Aus dem Physikalischen Institut der Chalmers Technischen Hochschule, Göteborg \\ (Z. Naturforschg. 11 a, 590-592 [1956]; eingegangen am 23. April 1956)
}

\begin{abstract}
Durch elektrolytische Überführung wurde an der Grenze zwischen geschmolzenem $\mathrm{LiBr}$ und $\mathrm{PbBr}_{2}$ ${ }^{7} \mathrm{Li}$ gegenüber ${ }^{6} \mathrm{Li}$ in $14^{1 / 3}$ Stunden um den Trennfaktor 3,63 angereichert. Der Masseneffekt war mit $-0,26 \pm 0,02$ beinahe doppelt so groß wie der früher für $\mathrm{LiCl}$ gefundene Wert.
\end{abstract}

Als erste Untersuchung des Isotopieeffektes in geschmolzenen Salzen haben Klemm und Mitarbeiter ${ }^{1}$ ${ }^{7} \mathrm{Li}$ an der Grenze zwischen $\mathrm{LiCl}$ und $\mathrm{PbCl}_{2}$ angereichert. Dabei wurde ein Masseneffekt von $\mu=-0,135$ gefunden. Dieser Wert ist später von Monse $^{2}$ bestätigt worden, der $\mu=-0,141$ fand. Innerhalb der Fehlergrenzen ist die Übereinstimmung beider Werte sehr gut. Auf Grund der allgemeinen Regelmäßigkeit des Masseneffektes in Halogenidschmelzen ${ }^{3}$ kann man erwarten, daß der Effekt in einem Bromid größer als in dem entsprechenden Chlorid ist. Dies ist für das Salzpaar $\mathrm{ZnCl}_{2}-\mathrm{ZnBr}_{2}$ experimentell bestätigt ${ }^{4}$. Bei einem leichten Element wie Lithium sollte aber die entsprechende Differenz ziemlich klein sein. Wir stellten uns die Aufgabe, diese Voraussage für das Salzpaar $\mathrm{LiCl}-\mathrm{LiBr}$ zu prüfen.

In dem in Abb. 1 wiedergegebenen Gefäß aus Supremaxglas wurden feste Stücke von (unter Vakuum schon umgeschmolzenem) $\mathrm{PbBr}_{2}$ und $\mathrm{LiBr}$ eingeführt und unter Vakuum geschmolzen. Wie üblich wollten wir das an der Kathode abgeschiedene Lithium in Salz zurückbilden. Vorversuche hatten aber gezeigt, daß die Halogenisierung des Metalls bei $\mathrm{LiBr}$ nicht so glatt wie bei $\mathrm{LiCl}$ verläuft. Um günstige Bedingungen für diese Reaktion zu schaffen, wurde die Kathode als Graphitrohr ausgebildet, durch das das Brom in die Schmelze hineinbrodelte. Das Rohr wurde am oberen Ende mittels Araldit mit einem Kugelschliff aus Pyrexglas verbunden. Sämtliche Verbindungen der Versuchsanordnung wurden mit solchen Schliffen versehen, denn z. B. Polyvinylchloridschläuche sind gegen Brom weniger beständig als gegen Chlor. Als Bromvorrat verwendeten wir wie früher ${ }^{4}$ einen Kolben, der jetzt in einem mit Tauchsieder und Kontaktthermometer ausgerüstetem Wasserbad angebracht war. Argon diente als Hilfsgas für die Überführung des Broms. Trotz dieser Anordnung war es leider nicht möglich, die Strömung des Broms konstant zu halten. Dies machte bei den Versuchen große

\footnotetext{
1 A. Klemm, H. Hintenberger u. P. Hoernes, Z. Naturforschg. 2 a, 245 [1947].

2 E. U. Monse, unveröffentlicht.
}

Schwierigkeiten. Der Überschuß des Gases wurde auf der anderen Seite des Elektrolysiergefäßes in einem mit Eis gekühlten Kolben gesammelt.

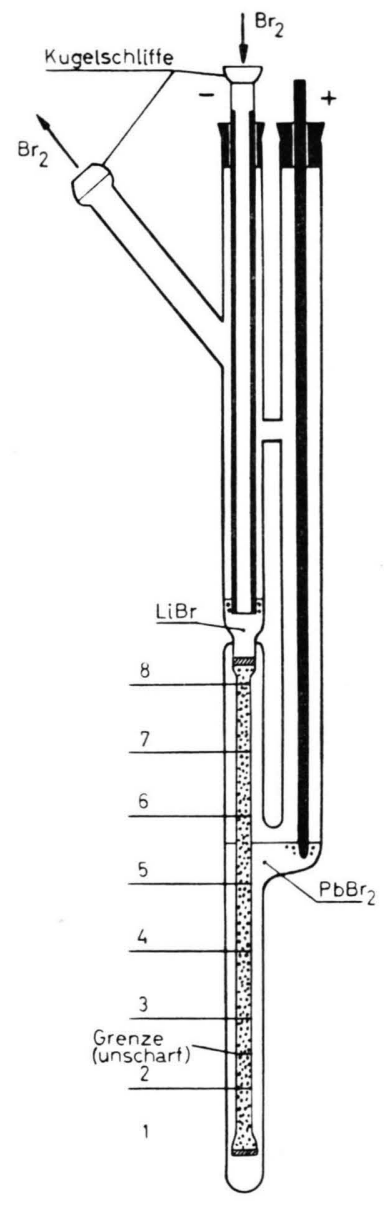

Abb. 1. Elektrolysiergefäß und Elektroden. Die Zerlegung des Trennrohres in 8 Proben ist schematisch angegeben.

Der Versuch wurde bei $590^{\circ} \mathrm{C}$ durchgeführt. Das Trennrohr war $25 \mathrm{~cm}$ lang und hatte $3,9 \mathrm{~mm}$ Durchmesser. In den ersten Stunden lag die Stromstärke

3 A. Klemm, Vortrag auf der Physikertagung in Wiesbaden. Sept. 1955.

4 A. Klemm, E. Lindholm u. A. Lundés, Z. Naturforschg. 7 a. 560 [1952]. 
wenig über $200 \mathrm{~mA}$. Nach etwa drei Stunden zeigte sich ein wenig Salz außen am Gefäß. Erst weitere $2^{1 / 2}$ Stunden später wurde der Sprung, der wie erwartet am Kathodenraum saß, sichtbar. Da ein gewisser Verlust von $\mathrm{LiBr}$ an der Kathode für den Mechanismus der Anreicherung im Trennrohr nicht gefährlich sein konnte, beschlossen wir, den Versuch mit möglichst hoher Stromstärke weiterzuführen, bis wegen der Undichtigkeit kein Kontakt zwischen Kathode und Schmelze mehr vorhanden sein würde. Es dauerte fast weitere 9 Stunden, ehe wir gezwungen waren, den Versuch abzubrechen.

Der Versuch lief $14^{1 / 3}$ Stunden und erbrachte, mit dem Kupfer-Coulometer gemessen, eine transportierte Ladung von $3,43 \mathrm{Ah}$. Die mittlere Stromstärke war also $239 \mathrm{~mA}$. Die Stromdichte wurde zu $4,4 \mathrm{~A} / \mathrm{cm}^{2}$ geschätzt.

Nach dem Erkalten wurde das Trennrohr in 8 Proben zerlegt, vgl. Abb. 1. Die gewogenen Proben wurden unter Verwendung eines Rückflußkühlers mit 30-proz. Ammoniumacetatlösung gekocht, bis alles Salz ausgelöst war. Das abfiltrierte Glas wurde gewogen und die gesamte Salzmenge dadurch erhalten. Durch wiederholtes Eindampfen mit $\mathrm{H}_{2} \mathrm{SO}_{4}$ wurde alles Salz in Sulfat überführt, wonach das $\mathrm{PbSO}_{4}$ gewogen wurde. Durch Differenzbildung erhielten wir die in Tab. 1 verzeichnete Menge von $\mathrm{LiBr}$ jeder Probe. Die Lösung von $\mathrm{Li}_{2} \mathrm{SO}_{4}$ wurde durch Fällung mit $\mathrm{Ba}\left(\mathrm{NO}_{3}\right)_{2}$ in eine Nitratlösung überführt, aus der das Barium durch Ausfällung mit $\left(\mathrm{NH}_{4}\right)_{2} \mathrm{CO}_{3}$ entfernt wurde. Nach Eindampfen mit $\mathrm{HNO}_{3}$ blieb nur $\mathrm{LiNO}_{3}$ übrig, das gewogen wurde, wodurch der Gehalt von $\mathrm{LiBr}$ noch einmal bestimmt wurde. Die Übereinstimmung der beiden Werte war für die meisten Proben gut. (Für die Proben 5 und 6 wurde die Überführung des Sulfates in Nitrat mit Ionenaustauscher gemacht.) Es ist zu bemerken, daß in sämtlichen Proben sowohl $\mathrm{LiBr}$ als $\mathrm{PbBr}_{2}$ vorhanden war. Diese Tatsache kann vielleicht dadurch erklärt werden, daß bei der Abkühlung noch geschmolzenes $\mathrm{PbBr}_{2}$ (Erstarrungspunkt $373^{\circ} \mathrm{C}$ ) in das schon erstarrte $\mathrm{LiBr}$ (Erstarrungspunkt $547^{\circ} \mathrm{C}$ ) eingedrungen ist, aber es ist auch möglich, daß wir keine scharfe Grenze während des Versuches hatten, obwohl das LiBr etwa 4-mal besser als $\mathrm{PbBr}_{2}$ leitet. (Leitfähigkeiten bei $590^{\circ} \mathrm{C}$ : $\mathrm{LiBr}$ $5,15 \Omega^{-1} \mathrm{~cm}^{-1}$ [1. c. ${ }^{5}$ ], $\mathrm{PbBr}_{2} 1,30 \Omega^{-1} \mathrm{~cm}^{-1}$ [l. c. ${ }^{6}$ ].) Der Zellenwiderstand schwankte während des Versuches, was auf eine Verschiebung des Salzes im Trennrohr hindeutet.

Es wurde bei der Analyse beobachtet, daß die Glaswände des Kathodenraumes und des Trennrohres, mit Ausnahme von Probe 1, grau gefärbt waren. Unter dem Mikroskop zeigte sich das Supremaxpulver der Proben 3-8 von der Schmelze stark angegriffen. Im Gegensatz zu $\mathrm{PbBr}_{2}$ greift also LiBr Glas stark an, eine Tatsache, die wir auch in anderem Zusammenhang beobachtet haben.

5 H. Bloom u. E. Heymann, Proc. Roy. Soc., Lond. A 188 , 395 [1947].

6 W. Biltz u. W. KLemm, Z.anorg. allg. Chem. 152, 267 [1926].
Das $\mathrm{LiNO}_{3}$ wurde beim A. E. R. E. in Harwell einer Analyse im Massenspektrometer unterworfen. Jede Probe wurde 10- bis 20 -mal gemessen. Das Verhältnis ${ }^{7} \mathrm{Li} /{ }^{6} \mathrm{Li}$ ist in Tab. 1 mit den mittleren Fehlern angegeben. Es wurde versucht, alle „Memory“-Effekte zwischen den verschiedenen Proben zu vermeiden. Die Probe 8, die zum größten Teil aus dem Kathodenraum stammt, ist als Normalprobe verwendet worden. Da die Probe 6 fast dieselbe Zusammensetzung hatte, wurde Probe 7 nicht gemessen. Ein Vergleich der gemessenen Häufigkeiten zeigt zwei Abweichungen gegenüber anderen Anreicherungsversuchen ${ }^{7}$. Erstens ist die Anreicherung in der Probe 1 viel kleiner als in den Proben 2 und 3. Probe 1 enthielt nur wenig $\mathrm{LiBr}$ in viel $\mathrm{PbBr}_{2}$, denn die unscharfe Grenze war in der Probe 2 gelegen. Das zweite unerwartete Ergebnis ist die große praktische Reichweite der Anreicherung von mehr als $10 \mathrm{~cm}$. Die effektive Selbstdiffusionskonstante wäre demnach mit etwa $50 \mathrm{~cm}^{2}$ pro Tag mehr als 10-mal größer gewesen als sonst für ähnliche Trennrohre gefunden ${ }^{7}$. (Unser Trennrohr war mit Supremax-Pulver Siebfraktion DIN $30-40$ gefüllt.) Diese Tatsache stützt die durch die chemische Analyse nahegelegte Vermutung, da $\beta$ entweder während des Versuches oder während der Erstarrung Verschiebungen im Trennrohr stattgefunden haben.

\begin{tabular}{|c|l|r|r|c|c|}
\hline $\begin{array}{c}\text { Probe } \\
\text { Nr. }\end{array}$ & $\begin{array}{c}\text { Länge } \\
\mathrm{mm}\end{array}$ & $\begin{array}{c}\mathrm{LiBr} \\
\mathrm{mg}\end{array}$ & $\begin{array}{c}\mathrm{PbBr}_{2} \\
\mathrm{mg}\end{array}$ & $\begin{array}{c}\text { Mole } \mathrm{LiBr} \\
{\mathrm{Mole} \mathrm{PbBr}_{2}}\end{array}$ & ${ }^{7} \mathrm{Li} /{ }^{6} \mathrm{Li}$ \\
\hline 1 & 50 & 51,47 & 2037,18 & 0,107 & $14,30 \pm 0,05$ \\
2 & 44,7 & 213,49 & 709,51 & 1,27 & $45,30 \pm 0,20$ \\
3 & 31 & 470,28 & 46,01 & 43,2 & $24,25 \pm 0,07$ \\
4 & 27,5 & 351,13 & 16,33 & 90,9 & $13,83 \pm 0,05$ \\
5 & 28 & 257,87 & 62,62 & 17,4 & $12,66 \pm 0,04$ \\
6 & 30 & 347,66 & 59,63 & 24,6 & $12,52 \pm 0,03$ \\
7 & 29 & 341,01 & 11,34 & 127 & - \\
8 & - & 4686,60 & 23,32 & 849 & $12,48 \pm 0,05$ \\
\hline
\end{tabular}

Tab. 1. Chemische Zusammensetzung und Häufigkeitsverhältnis der verschiedenen Proben.

In der üblichen Weise wurden die relative Differenz der Wanderungsgeschwindigkeiten und der Masseneffekt berechnet. Wir erhielten $\Delta w / w=0,04013$ und $\mu=-0,261$ (für $m=6,5$ ). Bei der vorliegenden Messung kann der Beitrag der Massenanalyse zum Gesamtfehler auf $4 \%$ geschätzt werden ${ }^{8}$, wenn wir 95\% Wahrscheinlichkeitsgrenzen angeben. Die Genauigkeit der chemischen Analyse ist an sich besser als $2 \%$, aber es war zu berücksichtigen, daß die Wägung von $\mathrm{LiNO}_{3}$ andere Gehalte an $\mathrm{LiBr}$ gab als die durch Differenzbildung erhaltenen in Tab. 1 eingetragenen Werte. Wegen Absorption bei den verschiedenen Fällungen sind die hier nicht angegebenen $\mathrm{LiNO}_{3}$-Wägungen als weniger zuverlässig zu

\footnotetext{
7 A. LundÉn, Dissertation Göteborg 1956, Tab. 1-13.

${ }^{8}$ 1. c. ${ }^{7}$, Kapitel 14.
} 
betrachten. Eine Berechnung mit diesen Werten gibt einen Masseneffekt $\mu=-0,255$, der nur um $2 \%$ von dem mit den genaueren LiBr-Gehalten berechneten $\mu$-Wert abweicht. Der Fehler der transportierten Ladung ist vernachlässigbar. Diese Diskussion zeigt, daß der Masseneffekt für Lithium in geschmolzenem Lithiumbromid

$$
\mu=-0,26 \pm 0,02
$$

ist, wobei der richtige Wert mit 95\% Wahrscheinlichkeit innerhalb der angegebenen Grenzen liegt.

Der erhaltene Masseneffekt ist um 86\% größer als derjenige des Lithiumchlorides $(\mu=-0,14)$. Der große Unterschied der beiden Masseneffekte kann vielleicht dadurch erklärt werden, daß $U^{+}$, die Selbstüberführungszahl des Lithiumions, in $\mathrm{LiBr}$ beträchtlich größer als in LiCl ist. Diese Zahl ist jedoch für geschmolzene Alkalihalogenide nicht bekannt. Eine weitere Diskussion soll anstehen, bis der Masseneffekt auch für LiJ gemessen ist.

Diese Arbeit ist von „Atomkommittén“ und „Chalmerska Forskningsfonden" finanziell unterstützt worden. Durch freundliches Entgegenkommen wurden die Massenanalysen beim A. E. R. E., Harwell, von Herrn Dr. G. H. Palmer gemacht, dem ich zu großem Dank verpflichtet bin. Ich danke auch Herrn Civiling. B. Stenemur für die Ausführung der chemischen Analyse und Herrn Professor N. Ryde für sein freundliches Interesse.

\title{
Untersuchungen über den Fadingmedhanismus bei Kernplatten (Agfa K-Platten) $)^{*}$
}

\author{
Von W. LohmanN \\ Aus dem Physikalischen Institut der Universität Freiburg (Brg.) \\ (Z. Naturforschg. 11 a, 592-598 [1956]; eingegangen am 21. März 1956)
}

\begin{abstract}
Mit der von Huang und Rhys ${ }^{1}$ entwickelten Theorie der Lichtabsorption wurde versucht, eine allgemein gültige Deutung des Fadingmechanismus zu geben. Die wesentlichste Reaktion beim Fadingprozeß ist die folgende: $\mathbf{A g} \rightarrow \mathrm{Ag}^{+}+$e. Die zu diesem Prozeß notwendige Energie wird der Wärmeenergie der Umgebung des Entwicklungszentrums entnommen. Bedingt durch die starke Kopplung von Gitter und Elektron findet ein Übergang von Wärmeenergie in Elektronenenergie statt. Dem Elektron muß dabei mindestens soviel Energie zugeführt werden, daß es energetisch in das Leitfähigkeitsband gelangen kann. Am wahrscheinlichsten sind dabei Prozesse, bei denen mehrere Gitterschwingungsquanten für einen Elektronenübergang gebraucht werden. Für einen großen Temperaturbereich ist die Übergangswahrscheinlichkeit proportional $\exp \left(-\varepsilon_{0} / k T\right)$. Ist $\varepsilon_{0}$ größer als $1 \mathrm{eV}$, so ist bei Zimmertemperatur die Übergangswahrscheinlichkeit vor allen Dingen durch den Effekt der quantenmechanischen Resonanz bedingt. Das ins Leitfähigkeitsband gehobene Elektron wird kurz darauf von angelagerten Wassermolekülen oder dgl. eingefangen, und auf diese Weise die Rückreaktion unterbunden. Daran schließen sich Sekundärreaktionen an, bei denen sich z. B. beim Wasser u. a. $\mathrm{HO}_{2}$ und $\mathrm{H}_{2} \mathrm{O}_{2}$ bilden, die ihrerseits sich schließlich wieder in Wasser und Sauerstoff umsetzen. Diese Überlegungen machen es verständlich, daß das Fading die beobachtete starke Abhängigkeit von der Temperatur $T$ zeigt, und daß es weiterhin stark von der Elektronenaffinität der zugefügten Substanzen abhängt. Im zweiten Teil der Arbeit werden einige Versuche beschrieben, die zur experimentellen Bestätigung obiger Theorie beitragen sollen.
\end{abstract}

\section{Theoretischer Teil}

Entwickelt man Kernplatten nicht sofort nach ihrer Exposition, so tritt ein Fading ein. Darunter versteht man die allmähliche zeitliche Abnahme der Korndichte einer Spur. Der Fadingkoeffizient $F$ ist

$$
F=\left(N_{0}-N\right) / N_{0} \text {. }
$$

$N_{0}$ ist die Kornzahl bei sofortiger Entwicklung, $N$ bei Entwicklung nach der Zeit $t$.

* Meinem hochverehrten Lehrer, Herrn Prof. Dr. E. BuchWALD, zum 70. Geburtstag gewidmet.
Dieser Effekt ist von verschiedenen Autoren untersucht worden. Es hat sich gezeigt, daß im Vakuum nur geringes Fading auftritt. Dies berechtigt zu der Annahme, daß der Effekt zum größten Teil durch Bestandteile der Luft hervorgerufen wird. Der restliche Anteil ist nach Beiser ${ }^{2}$ durch thermische Ejektion der Elektronen der Silberentwicklungszentren bedingt. Die Silberionen verlassen dann das Entwicklungszentrum, wodurch dessen Größe reduziert

1 Huang u. Rhys, Proc. Roy. Soc., Lond. A 204, 406 [1950]. 2 A. Beiser, Rev. Mod. Phys. 24, (4) 273 [1952]. 